\title{
Fatty acid profiles in the gonads of the sea urchin Strongylocentrotus droebachiensis on natural algal diets
}

\author{
Jennifer R. Kelly ${ }^{1, *}$, Robert E. Scheibling ${ }^{1}$, Sara J. Iverson ${ }^{1}$, Patrick Gagnon ${ }^{2}$ \\ ${ }^{1}$ Department of Biology, Dalhousie University, Halifax, Nova Scotia B3H 4J1, Canada \\ ${ }^{2}$ Ocean Sciences Centre, Memorial University of Newfoundland, St. John's, Newfoundland and Labrador A1C 5S7, Canada
}

\begin{abstract}
We examined fatty acid (FA) compositions of gonads of the green sea urchin Strongylocentrotus droebachiensis collected from a grazing aggregation (front) at the edge of a kelp bed and from barrens dominated by coralline algae, and those of urchins fed single algal diets in the laboratory. We compared these gonad FAs with those of the algal diets, which represented known urchin food sources in rocky subtidal habitats. Gonads of urchins collected from both wild habitats, and of urchins fed kelp Saccharina longicruris in the laboratory, contained more lipid than did urchins fed single diets of barrens macroalgae (Agarum clathratum, coralline red algae or Desmarestia viridis). Substantial biosynthesis of non-methylene interrupted dienes and other FAs by urchins markedly affected their overall FA signatures. Although the FA compositions of gonads of laboratory-fed urchins did not clearly correspond with those of their diets, 3 clusters of urchins were distinguished in multivariate space using multidimensional scaling (MDS): (1) urchins fed single diets of barrens macroalgae in the laboratory; (2) urchins fed S. longicruris in the laboratory or collected from the grazing front; and (3) urchins from the barrens. Characteristics of FA signatures found in urchins from the barrens suggested the occurrence of benthic diatoms in their diet. Our results indicate that, while the FA signatures of urchin gonads are affected by diet and can be used to differentiate feeding groups of urchins in the laboratory and field, significant de novo biosynthesis and/or modification of FAs precludes correspondence of urchin FAs to those of their algal diets.
\end{abstract}

KEY WORDS: Fatty acid - Sea urchin - Strongylocentrotus droebachiensis · Macroalgae · Dietary tracer . Food web

Resale or republication not permitted without written consent of the publisher

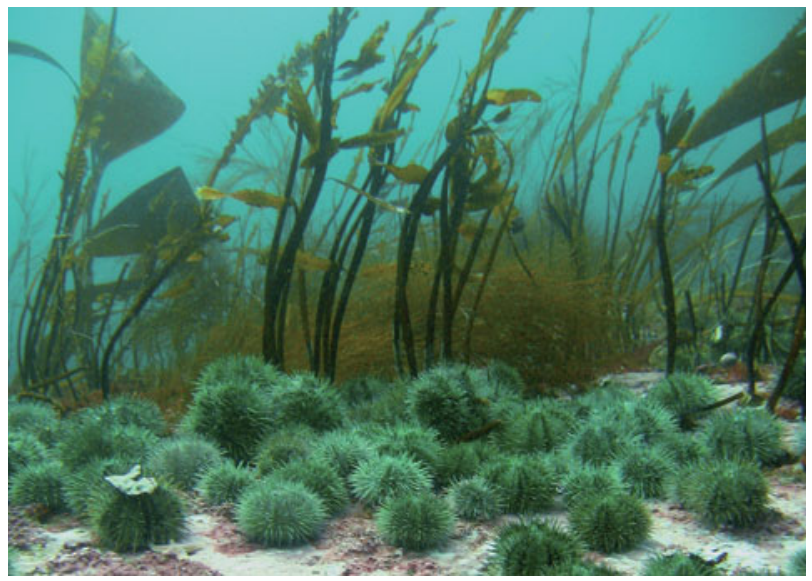

Population dynamics in sea urchins Strongylocentrotus droebachiensis mediate ecosystem phase shifts between kelp beds and coralline barrens off Nova Scotia.

Photo: R. E. Scheibling

\section{INTRODUCTION}

Fatty acid (FA) analysis is a powerful ecological tool for discerning trophic relationships in terrestrial and aquatic ecosystems (Dalsgaard et al. 2003, Budge et al. 2006, Iverson 2009). Unlike traditional methods of studying diet, such as gut content analysis, FA profiles of consumers can provide information that is neither biased towards food items with hard parts, nor representative of only the last meal (e.g. Iverson et al. 1997, 2004, Budge et al. 2002, 2006). FAs were first used as dietary tracers in bottom-up studies of marine zooplankton (reviewed in Dalsgaard et al. 2003). These 
studies, and subsequent bottom-up studies of marine food webs, were facilitated by the disparate FA profiles of various classes of primary producers. Diatoms, dinoflagellates, bacteria, land plants and macroalgae can be identified by either unique classes of FAs or characteristic ratios of selected FAs (Sargent et al. 1988, Parrish et al. 2000). Generally, these FAs are passed up the food chain in a predictable manner such that a consumer's FA composition will be strongly influenced by that of its diet (e.g. Graeve et al. 1994, 2002, Rooker et al. 2006) and this potentially allows estimation of dietary composition, at least in higher order consumers (Iverson et al. 2004, Iverson 2009).

In marine systems, certain FAs can be used as indicators for the general type of primary producer in which they originated (Sargent et al. 1988). These marker FAs, when found in consumer tissues, can be used to characterize some food webs. For example, mangrove and seagrass ecosystems are well suited to this type of marker analysis, as these inputs of primary production are indistinguishable by the presence of long-chain FAs unique to angiosperms (Nichols et al. 1986, Kharlamenko et al. 2001, Alfaro et al. 2006, Hall et al. 2006, Meziane et al. 2006). In systems with several taxonomically similar primary producers, a higher level of resolution may be necessary to understand trophic relationships. In general, marine macroalgae can be identified to species level using FA analysis (Graeve et al. 2002, Khotimchenko et al. 2002, Nelson et al. 2002, Khotimchenko 2003), and further distinction among macroalgal sources of primary production may be possible at higher trophic levels, such as in fish consumers (Rooker et al. 2006).

In vertebrate predators, the array of FAs (i.e. the fatty acid signature; Iverson 1993) in fat stores indicates specific prey consumption (Kirsch et al. 1998, Iverson et al. 2004, 2007, Nordstrom et al. 2008). However, the same taxonomic resolution may not be attainable with certain invertebrate herbivores, which are capable of greatly modifying or biosynthesizing FAs (Dalsgaard et al. 2003, Iverson 2009). Numerous studies have used field measurements of marker FAs in invertebrate herbivores to evaluate diet (Bottino 1974, Hayashi \& Takagi 1977, Takagi et al. 1980) or to trace sources of primary production (Nichols et al. 1986, Pond et al. 1995, Kharlamenko et al. 2001, Reuss \& Poulsen 2002, Alfaro et al. 2006, Meziane et al. 2006). Others have studied the effects of known diets on consumer FA composition (Graeve et al. 1994, Pantazis et al. 2000, Castell et al. 2004, Hall et al. 2006). Few studies have compared the FA composition of such organisms fed known diets in the laboratory with those collected from the wild in order to infer diet of wild collected animals (Cook et al. 2000). The use of FAs as dietary tracers in invertebrate grazers requires controlled feeding experiments, employing naturally occurring diets, before the technique can be applied in the field.

The green sea urchin Strongylocentrotus droebachiensis is an ideal model organism to study the effects of dietary FAs on an invertebrate herbivore because it is a generalist grazer whose feeding ecology is well known from field studies (reviewed by Scheibling \& Hatcher 2007). In the rocky subtidal ecosystem of Nova Scotia, the urchin plays a key role in driving phase shifts between 2 states: kelp beds (mainly Saccharina longicruris and Laminaria digitata) with a low urchin density, and barrens, dominated by urchins and encrusting coralline algae (Phymatolithon spp., Lithothamnion glaciale, Clathromorphum circumscriptum) (Scheibling 1986, Lauzon-Guay \& Scheibling 2007). Dense feeding aggregations of urchins (fronts) destructively graze kelp beds, while more scattered populations of urchins consume coralline algae and microalgal films in barrens (Johnson \& Mann 1982, Scheibling 1986, Scheibling et al. 1999, Lauzon-Guay \& Scheibling 2007). Urchins in kelp beds and feeding fronts have a higher nutritional condition than do those in barrens, and develop a larger gonadal mass (Meidel \& Scheibling 1998).

Most lipid storage in sea urchins occurs in the gonads, and several studies have compared FA signatures of urchin gonads on kelp diets with those of urchins on a variety of artificial diets in fisheries and aquaculture applications (Cook et al. 2000, LiyanaPathirana et al. 2002, Castell et al. 2004). While these studies have shown that FA compositions of urchins resemble those of their diets to some extent, the experimental diets were markedly different from each other and bore little resemblance to food normally encountered by urchins in the wild (i.e. experimental diets were composed of different nutrients and/or had higher dry matter and fat contents). Our study evaluates the use of FAs as dietary tracers at the base of naturally occurring rocky subtidal food webs from macroalgae to sea urchins. To determine the degree to which dietary FAs are deposited directly into urchin tissues, we compared FA compositions of 4 natural algal diets to those of gonadal tissue produced by urchins fed each diet. We also examined the FA compositions of urchins collected from 2 subtidal areas, a grazing front along the edge of a kelp bed and an adjacent urchin barrens, to compare these with the FA compositions of urchins fed the 4 algal diets in the laboratory.

\section{MATERIALS AND METHODS}

Feeding experiment. Our experiment was part of a broader study to examine growth, reproduction and physical performance of Strongylocentrotus droebachi- 
ensis fed dominant species of macroalgae from urchin barrens, using kelp Saccharina longicruris, a species absent in barrens but resulting in high rates of somatic and gonadic growth (Scheibling \& Hatcher 2007), as a control. Urchins were collected by divers in February 2006 from a grazing front at the lower margin of a kelp bed (S. longicruris and Laminaria digitata) at Splitnose

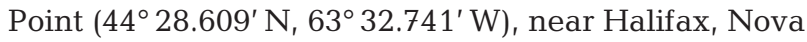
Scotia, Canada. Urchins were maintained in $75 \mathrm{l}$ glass aquaria supplied with flowing seawater and fed $50 \mathrm{~g}$ of kelp ( $S$. longicruris) once or twice weekly (ad libitum) for $12 \mathrm{wk}$, and then fasted for $2 \mathrm{wk}$ until the start of the experiment. On 9 June, 20 urchins were placed in each of 5 replicate aquaria for each of 4 algal diets: (1) a kelp of low preference rank (Agarum clathratum), (2) crustose coralline algae (Phymatolithon spp., Lithothamnion glaciale, Clathromorphum circumscriptum), (3) a chemically defended brown alga (Desmarestia viridis) and (4) a highly preferred kelp ( $S$. longicruris). Algae were collected by divers from Splitnose Point as needed throughout the experiment; A. clathratum, $D$. viridis and $S$. longicruris were cut off above the holdfast, and corallines were chipped off the seabed using hammers and chisels. Algae were maintained in aquaria supplied with flowing seawater. Urchins were fed each diet ad libitum (once or twice weekly) for $14 \mathrm{wk}$. Algal rations were blotted with paper towel for 40 to $60 \mathrm{~s}$ and weighed before being added to urchin aquaria, with the exception of $D$. viridis, which was blotted more rapidly (10 to $15 \mathrm{~s}$ ) to avoid release of sulfuric acid caused by contact with air. Uneaten pieces of algae were blotted, weighed and discarded before fresh rations were added so that consumption could be estimated for each feeding interval. At the end of the experiment, 5 urchins per tank were randomly selected for FA analysis.

In November 2007, 20 urchins were collected from the grazing front at Splitnose Point and another 20 from the coralline algae dominated barrens about $10 \mathrm{~m}$ offshore of the front. Kelp biomass at the grazing front consisted of approximately equal proportions of Saccharina longicruris and Laminaria digitata (LauzonGuay \& Scheibling 2007). Urchins were held in flowing seawater tanks for $24 \mathrm{~h}$ before being sacrificed for FA analysis.

Lipid extraction. Algae were blotted to remove excess water and visually examined for epibionts, which were removed prior to processing. Whole thalli of Desmarestia viridis were homogenized using a handheld immersion blender; kelps (Saccharina longicruris, Agarum clathratum) were coarsely chopped and homogenized into a paste using a mortar and pestle; fragments of coralline algae (chipped or pried off the bottom with a diver's knife) were crushed with mortar and pestle. Samples of each alga weighing $9.0 \mathrm{~g}(\mathrm{n}=2$ for coralline algae and $D$. viridis, $\mathrm{n}=3$ for $A$. clathratum and $S$. longicruris), each from a different thallus where applicable, were weighed before adding $20 \mathrm{ml}$ chloroform and $10 \mathrm{ml}$ methanol containing $0.01 \%$ butylated hydroxytoluene (BHT) as an antioxidant.

Gonads were excised from 10 randomly selected urchins per diet treatment and stored at $-20^{\circ} \mathrm{C}$ for 30 to $90 \mathrm{~d}$ prior to lipid extraction. For lipid extraction, $1.5 \mathrm{~g}$ of gonad from each urchin was manually homogenized with $20 \mathrm{ml}$ chloroform and $10 \mathrm{ml}$ methanol containing $0.01 \%$ BHT.

Lipid extraction protocols were modified from Folch et al. (1957). The samples in solvent (urchin gonad or algae) were filtered (Fisher P8 filter paper) to remove residual tissue before adding $7 \mathrm{ml}$ of $5 \% \mathrm{NaCl}$. Samples were shaken and then centrifuged for $20 \mathrm{~min}$. The lipid-containing lower phase was filtered through $\mathrm{NaSO}_{4}$ and evaporated to dryness under nitrogen at 25 to $30^{\circ} \mathrm{C}$ in a pre-weighed boiling tube. The boiling tube was re-weighed to calculate the amount of crude lipid for each sample.

Fatty acid methyl ester (FAME) preparation and analysis. To prepare lipids for FA analysis, crude lipid was dissolved in $1.5 \mathrm{ml}$ methylene chloride with $0.01 \%$ BHT and subjected to acidic transesterification $\left(\mathrm{H}_{2} \mathrm{SO}_{4}\right.$ in methanol) to produce FA methyl esters (FAME), as described in Budge et al. (2006).

FAME were purified to remove proteins and pigments using thin layer chromatography (TLC). FAME were dissolved in hexane and spotted onto silica TLC plates (Sigma) and developed in a TLC chamber with 90:10:1 petroleum ether : ethyl ether : acetic acid for approximately $45 \mathrm{~min}$. Plates were sprayed with 2,4 dichlorofluorescein and viewed under UV light to mark the FAME containing band. The FAME containing band was then scraped from the silica plate and transferred to a funnel packed with silane treated glass wool. Chloroform containing $0.01 \%$ BHT was used to dissolve FAME into a pre-weighed tube. The sample was evaporated to dryness under nitrogen at 25 to $30^{\circ} \mathrm{C}$ and hexane was added to a final concentration of $50 \mathrm{mg} \mathrm{ml}^{-1}$. Samples were stored at $-20^{\circ} \mathrm{C}$ prior to analysis.

Duplicate samples of FAME were analyzed and FAs identified using temperature programmed gas chromatography (Iverson et al. 1997, 2004, Budge et al. 2006).

Statistical methods. Lipid content of urchin gonads (\% wet wt) was compared across the 6 treatments using 1-way ANOVA. Lipid content data met ANOVA assumptions of normality and homoscedasticity, and were not transformed prior to analysis. Post hoc pairwise comparisons were made using Bonferroni adjustments for multiple comparisons.

Samples with a FAME weight $\geq 0.005$ g (64 out of 80 samples; 4 samples from each diet group of laboratory 
fed urchins were eliminated due to low FAME weight) were used in multivariate analyses. Only FAs with an overall mean contribution $\geq 0.1 \%$ were used and FA composition was normalized to $100 \%$ for all samples.

Levels of selected FAs were compared among algal species using 1-way ANOVA following tests to confirm that data met ANOVA assumptions. Post hoc pairwise comparisons were made with Bonferroni adjustments. FA compositions were compared among algae, and between urchin gonads and their respective diets using analysis of similarity (ANOSIM) on Bray-Curtis distances (Clarke \& Warwick 1994) of untransformed data. FA composition of urchin gonads was compared across the 6 diet and habitat groups using multidimensional scaling (MDS) and ANOSIM on Bray-Curtis distances (Clarke \& Warwick 1994). Homogenous clusters of diet and habitat groups were determined using pairwise comparisons in ANOSIM (Bonferroni adjusted $\alpha=$ 0.05). Urchin gonad data were then pooled according to these homogenous clusters, and similarity percentage (SIMPER) analysis was conducted on the clusters to determine the contribution of each FA to the average Bray-Curtis similarity within each cluster, and to the average Bray-Curtis dissimilarity among groups (Clarke \& Warwick 1994). All multivariate analyses were conducted using PRIMER 5 software (Clarke \& Warwick 1994).

\section{RESULTS}

\section{Algal consumption by urchins}

Over the course of the $14 \mathrm{wk}$ feeding experiment, mean \pm SE consumption rates per urchin (calculated on a per aquarium basis, $\mathrm{n}=5$ aquaria per algal diet) on the 4 algal diets were: $0.43 \pm 0.010 \mathrm{~g} \mathrm{~d}^{-1}$ on Agarum clathratum, $0.013 \pm 0.004 \mathrm{~g} \mathrm{~d}^{-1}$ of coralline algae, $0.47 \pm$ $0.008 \mathrm{~g} \mathrm{~d}^{-1}$ on Desmarestia viridis and $1.56 \pm 0.054 \mathrm{~g} \mathrm{~d}^{-1}$ on Saccharina longicruris.

\section{Lipid content and fatty acid composition of algae}

The 4 algal diets (Agarum clathratum, coralline algae, Desmarestia viridis, and Saccharina longicruris) did not differ in lipid content $\left(F_{3,6}=0.902, \mathrm{p}=0.493\right.$; Table 1), but differed in FA composition (ANOSIM, $\mathrm{p}<0.001$; Table 1). The saturated FA 16:0 was a major component of all algal diets, constituting between 13.4 and $25.0 \%$ of total FAs. Polyunsaturates 20:4n-6 and 20:5n-3 also were major components of all algal diets, making up 3.9 to $15.1 \%$ and 8.8 to $27.9 \%$ of total FAs, respectively. A. clathratum differed from other algal diets in having relatively high levels of 16:4n-1, and relatively low levels of 20:4n-6 and 20:5n-3. Coralline algae and $A$. clathratum both contained higher levels of $16: 0$ and 22:5n-3 than did D. viridis or S. longicruris. $S$. longicruris and $D$. viridis contained higher levels of 18:4n-3 than did coralline algae or A. clathratum. Relative to all other algal diets, corallines were rich in 16:0 and 20:5n-3 (ANOVA, $p<0.01$ for all comparisons among algae).

\section{Lipid content and fatty acid composition of urchin gonads}

Lipid content of urchin gonads ranged from $0.7 \%$ (fed Desmarestia viridis) to $9.2 \%$ (fed Saccharina longicruris). Gonad lipid content differed significantly among groups of laboratory fed and wild collected urchins $\left(F_{5,78}=9.791, \mathrm{p}<0.001 ;\right.$ Fig. 1$)$. Gonads of urchins fed coralline algae and $D$. viridis in the laboratory had significantly lower lipid content than did those fed $S$. longicruris or collected from kelp bed or barrens habitats. Gonad lipid content of urchins fed Agarum clathratum did not differ significantly from that of other groups.

Urchin gonads contained all FAs in the respective algal diet, as well as a number of others that were absent from or present only in trace amounts in the diet, but contributed markedly to the overall FA signature of the gonads (Table 1). The FAs found only in urchins were primarily 20:1n-9, 20:1n-7, 22:1n-9 and several non-methylene interrupted dienes $(20: 2 \Delta 5,11$; $20: 2 \Delta 5,13 ; 20: 2 \Delta 5,11$ and 20:2 $\Delta 5,13)$. Several other FAs were found in urchin gonads at higher levels than in their diet (e.g. 14:0, 16:1n-7). Overall, the FA signatures of urchin gonads did not resemble those of their respective diets (ANOSIM, $p<0.04$ for all diets; Table 1). However, urchins were separated into 3 homogenous clusters using MDS and ANOSIM: (1) urchins fed Agarum clathratum, coralline algae or Desmarestia viridis in the laboratory; (2) wild collected

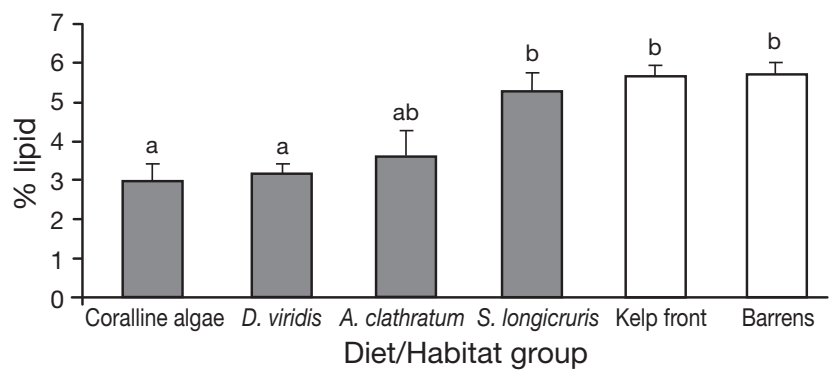

Fig. 1. Strongylocentrotus droebachiensis. Lipid content of gonads from laboratory fed (shaded bars) and wild (unshaded bars) sea urchins. Data are mean lipid weight expressed as percent of gonad wet weight + SE. Letters above bars that are not in common indicate lipid contents that are significantly different $(p<0.05)$ 
Table 1. Relative fatty acid composition of algae used in feeding experiments and of sea urchin Strongylocentrotus droebachiensis gonads from laboratory fed and wild specimens. Lipid content values are mean percent of total wet weight \pm SE. Fatty acid values are mean percent of total fatty acids \pm SE of all fatty acids with overall mean concentration $\geq 0.1 \%$

\begin{tabular}{|c|c|c|c|c|c|c|c|c|c|c|}
\hline & \multirow[b]{2}{*}{$\begin{array}{l}\text { Agarum } \\
\text { clathratum }\end{array}$} & \multicolumn{3}{|c|}{ Algae } & \multicolumn{4}{|c|}{ - Laboratory urchins } & \multicolumn{2}{|c|}{ Wild urchins } \\
\hline & & $\begin{array}{l}\text { Coralline } \\
\text { algae }\end{array}$ & $\begin{array}{c}\text { Desmarestia } \\
\text { viridis }\end{array}$ & $\begin{array}{l}\text { Saccharina } \\
\text { longicruris }\end{array}$ & $\begin{array}{c}\text { Fed } A . \\
\text { clathratum }\end{array}$ & $\begin{array}{l}\text { Fed coral- } \\
\text { line algae }\end{array}$ & $\begin{array}{l}\text { Fed } D \text {. } \\
\text { viridis }\end{array}$ & $\begin{array}{c}\text { Fed } S . \\
\text { longicruris }\end{array}$ & Barrens & Kelp front \\
\hline $\begin{array}{l}\mathrm{n} \\
\% \text { Lipid }\end{array}$ & $\begin{array}{c}3 \\
0.08 \pm 0.03\end{array}$ & $\begin{array}{c}2 \\
0.04 \pm 0.03\end{array}$ & $\begin{array}{c}2 \\
0.09 \pm 0.01\end{array}$ & $\begin{array}{c}3 \\
0.02 \pm 0.01\end{array}$ & $\begin{array}{c}5 \\
3.62 \pm 0.62\end{array}$ & $\begin{array}{c}6 \\
2.95 \pm 0.49\end{array}$ & $\begin{array}{c}9 \\
3.11 \pm 0.31\end{array}$ & $\begin{array}{c}11 \\
5.16 \pm 0.60\end{array}$ & $\begin{array}{c}12 \\
5.62 \pm 032\end{array}$ & $\begin{array}{c}21 \\
5.69 \pm 0.34\end{array}$ \\
\hline 13:0 & - & $0.07 \pm 0.02$ & $0.07 \pm 0.01$ & $0.09 \pm 0.02$ & $0.23 \pm 0.02$ & $0.28 \pm 0.04$ & $0.24 \pm 0.01$ & $0.16 \pm 0.01$ & $0.06 \pm 0.01$ & $0.08 \pm 0.01$ \\
\hline iso14:0 & $0.11 \pm 0.01$ & $0.82 \pm 0.29$ & $0.53 \pm 0.29$ & $0.22 \pm 0.15$ & $0.03 \pm 0.00$ & $0.05 \pm 0.00$ & $0.06 \pm 0.00$ & $0.03 \pm 0.00$ & $0.11 \pm 0.01$ & $0.05 \pm 0.00$ \\
\hline $14: 0$ & $2.88 \pm 0.19$ & $2.67 \pm 0.41$ & $5.53 \pm 0.17$ & $6.75 \pm 0.49$ & $10.39 \pm 0.42$ & $9.62 \pm 0.52$ & $8.55 \pm 0.12$ & $13.31 \pm 0.74$ & $9.66 \pm 0.33$ & $12.18 \pm 0.38$ \\
\hline $14: 1 n-9$ & - & $0.24 \pm 0.23$ & - & $0.01 \pm 0.01$ & $0.20 \pm 0.01$ & $0.16 \pm 0.01$ & $0.17 \pm 0.00$ & $0.17 \pm 0.01$ & $0.14 \pm 0.00$ & $0.18 \pm 0.01$ \\
\hline $14: 1 n-5$ & $0.06 \pm 0.02$ & $0.26 \pm 0.06$ & $0.11 \pm 0.08$ & $0.10 \pm 0.01$ & $1.58 \pm 0.18$ & $1.30 \pm 0.17$ & $0.96 \pm 0.02$ & $1.57 \pm 0.17$ & $0.74 \pm 0.05$ & $1.55 \pm 0.13$ \\
\hline & $0.08 \pm 0.02$ & $0.62 \pm 01.11$ & $0.17 \pm 0.04$ & $0.13 \pm 0.02$ & $0.14 \pm 0.01$ & $0.14 \pm 0.02$ & $0.18 \pm 0.00$ & $0.13 \pm 0.02$ & $0.45 \pm 0.03$ & $0.15 \pm 0.01$ \\
\hline anti15:0 & $0.01 \pm 0.01$ & $0.31 \pm 0.00$ & - & - & $0.06 \pm 0.01$ & $0.08 \pm 0.01$ & $0.08 \pm 0.00$ & $0.06 \pm 0.01$ & $0.24 \pm 0.02$ & $0.07 \pm 0.01$ \\
\hline $15: 0$ & $0.26 \pm 0.05$ & $0.39 \pm 0.00$ & $0.14 \pm 0.01$ & $0.25 \pm 0.03$ & $0.59 \pm 0.05$ & $0.63 \pm 0.05$ & $0.62 \pm 0.01$ & $0.53 \pm 0.02$ & $0.83 \pm 0.04$ & $0.43 \pm 0.02$ \\
\hline $16: 0$ & $18.22 \pm 0.31$ & $24.95 \pm 1.79$ & $13.53 \pm 0.05$ & $13.40 \pm 1.28$ & $11.72 \pm 0.32$ & $11.87 \pm 0.26$ & $12.39 \pm 10$ & $14.17 \pm 0.28$ & $13.14 \pm 0.30$ & $14.31 \pm 0.27$ \\
\hline $16: 1 n-11$ & $1.84 \pm 0.06$ & $3.70 \pm 0.27$ & $1.37 \pm 1.28$ & $1.39 \pm 0.02$ & $0.28 \pm 0.02$ & $0.33 \pm 0.02$ & $0.36 \pm 0.01$ & $0.25 \pm 0.02$ & $0.42 \pm 0.03$ & $0.34 \pm 0.02$ \\
\hline $16: 1 n-9$ & $0.39 \pm 0.13$ & $23 \pm 0.09$ & $1.56 \pm 1.35$ & $0.13 \pm 0.02$ & $0.27 \pm 0.01$ & $0.26 \pm 0.02$ & $0.28 \pm 0.00$ & $0.21 \pm 0.01$ & $0.36 \pm 0.01$ & $0.24 \pm 0.01$ \\
\hline $16: 1 n-7$ & $5.66 \pm 1.89$ & $72 \pm 0.47$ & $2.61 \pm 0.15$ & $2.88 \pm 0.35$ & $5.96 \pm 0.32$ & $5.94 \pm 0.32$ & $5.83 \pm 0.11$ & & $5.94 \pm 0.27$ & \\
\hline $16: 1$ & $0.76 \pm 0.32$ & $0.28 \pm 0.01$ & $4.53 \pm 0.03$ & $0.38 \pm 0.03$ & $3.71 \pm 0.25$ & $3.46 \pm 0.30$ & $2.62 \pm 0.05$ & $3.13 \pm 0.18$ & $2.18 \pm 0.15$ & $3.66 \pm 0.18$ \\
\hline $16: 2 n-6$ & $0.22 \pm 0.04$ & $0.02 \pm 0.01$ & $0.07 \pm 0.00$ & $0.34 \pm 0.04$ & $0.06 \pm 0.00$ & $0.05 \pm 0.00$ & $0.06 \pm 0.00$ & $0.11 \pm 0.01$ & $0.14 \pm 0.01$ & $0.07 \pm 0.00$ \\
\hline iso17:0 & $0.03 \pm 0.01$ & $1.25 \pm 0.90$ & - & - & $7 \pm 0.01$ & $0.08 \pm 0.02$ & $0.10 \pm 0.00$ & $7 \pm 0.01$ & $0.41 \pm 0.04$ & 0.01 \\
\hline $16: 2 n-4$ & - & $0.04 \pm 0.01$ & - & $0.01 \pm 0.00$ & $8 \pm 0.03$ & $0.25 \pm 0.04$ & $25 \pm 0.01$ & $0.14 \pm 0.02$ & $0.20 \pm 0.01$ & $12 \pm 0.01$ \\
\hline $16: 3 n-6$ & $1.99 \pm 0.86$ & $0.83 \pm 0.12$ & $0.21 \pm 0.01$ & & & & $0.11 \pm 0.00$ & & $0.54 \pm 0.08$ & \\
\hline $17: 0$ & $09 \pm 0.02$ & $27 \pm 0.02$ & $0.05 \pm 0.01$ & & & & & & $0.18 \pm 0.01$ & \\
\hline $16: 3 n-4$ & $1.83 \pm 0.41$ & $0.15 \pm 0.05$ & $0.07 \pm 0.01$ & $0.29 \pm 0.02$ & $0.09 \pm 0.03$ & $0.08 \pm 0.01$ & $9 \pm 0.00$ & $0 \pm 0.01$ & $0.81 \pm 0.01$ & $4 \pm 0.02$ \\
\hline $17: 1$ & $0.75 \pm 0.70$ & $0.12 \pm 0.00$ & $0.03 \pm 0.00$ & $0.04 \pm 0.00$ & $0.10 \pm 0.08$ & & $1 \pm 0.00$ & & $0.14 \pm 0.29$ & \\
\hline $16: 4 n-3$ & - & - & - & - & & & & & $0.16 \pm 0.06$ & \\
\hline $16: 4 n-1$ & $6.13 \pm 1.45$ & $0.08 \pm 0.01$ & $0.30 \pm 0.01$ & $0.56 \pm 0.10$ & 05 & 0.04 & $9 \pm 0.01$ & 0.1 & $1.76 \pm 0.02$ & 0.05 \\
\hline $18: 0$ & $3.95 \pm 1.04$ & & $8 \pm 1.18$ & & & & & & $1.47 \pm 0.13$ & \\
\hline $18: 1 n-13$ & - & $17 \pm 0.10$ & $6 \pm 0.03$ & & & & & & $0.40 \pm 0$ & \\
\hline $18: 1 n-9$ & $11.32 \pm 4.67$ & $04 \pm 0.85$ & $5.25 \pm 0.04$ & $5.33 \pm 0.29$ & $2.96 \pm 0.14$ & $2.71 \pm 0.15$ & $2.90 \pm 0.04$ & $2.59 \pm 0.09$ & $2.10 \pm 0.02$ & $2.33 \pm 0.11$ \\
\hline $18: 1 n-7$ & $0.32 \pm 0.02$ & $70 \pm 0.15$ & $0.37 \pm 0.05$ & $0.34 \pm 0.06$ & $3.66 \pm 0.11$ & $3.94 \pm 0.16$ & $3.51 \pm 0.04$ & $3.30 \pm 0.07$ & $3.60 \pm 0.08$ & $3.88 \pm 0.07$ \\
\hline $18: 1 n-5$ & $10 \pm 0.01$ & \pm 0.05 & $0.32 \pm 0.01$ & & & & & & 05 & \\
\hline $18: 2 n-7$ & $0.06 \pm 0.01$ & $0.02 \pm 0.02$ & - & $0.02 \pm 0.01$ & $1.08 \pm 0.05$ & $1.05 \pm 0.18$ & $1.09 \pm 0.04$ & $1.05 \pm 0.09$ & $0.69 \pm 0.01$ & $0.98 \pm 0.07$ \\
\hline $18: 2 n-6$ & $7.39 \pm 3.05$ & $2.82 \pm 1.14$ & $4.56 \pm 0.04$ & $6.27 \pm 0.06$ & $1.04 \pm 0.08$ & $0.97 \pm 0.05$ & $0.96 \pm 0.02$ & $1.19 \pm 0.04$ & $1.23 \pm 0.05$ & $1.10 \pm 0.05$ \\
\hline $18: 2 n-4$ & $0.64 \pm 0.26$ & \pm 0.02 & $1 \pm 0.00$ & $0.03 \pm 0$ & & .01 & $=0.00$ & 0.01 & 01 & 0.01 \\
\hline $18: 3 n-6$ & $0.42 \pm 0.13$ & $23 \pm 0.05$ & $0.74 \pm 0.03$ & $0.91 \pm 0.06$ & $0 \pm 0.02$ & $0.17 \pm 0.01$ & $0 \pm 0.00$ & $7 \pm 0.01$ & $0.44 \pm 0.03$ & $3 \pm 0.01$ \\
\hline $18: 3 n-4$ & $0.10 \pm 0.03$ & $0.02 \pm 0.01$ & - & - & $0.14 \pm 0.02$ & $0.15 \pm 0.01$ & $0.16 \pm 0.00$ & $0.12 \pm 0.01$ & $0.33 \pm 0.03$ & $0.12 \pm 0.01$ \\
\hline $18: 3$ & $4 \pm 0.47$ & \pm 0.07 & $11.33 \pm 0.3$ & $5.95 \pm 1$ & & & & & 05 & 0.07 \\
\hline $18: 3 n-1$ & $0.98 \pm 0.37$ & $0.13 \pm 0.10$ & $0.12 \pm 0.03$ & $0.33 \pm 0.03$ & $1 \pm 0.01$ & $2 \pm 0.01$ & $3 \pm 0.00$ & $1 \pm 0.01$ & $0.10 \pm 0.02$ & \pm 0.00 \\
\hline $18: 4 n-3$ & $2.53 \pm 0.25$ & $0.39 \pm 0.00$ & $15.64 \pm 0.57$ & $14.79 \pm 2.69$ & $2.66 \pm 0.23$ & $2.81 \pm 0.29$ & $2.25 \pm 0.03$ & $0 \pm 0.16$ & $2.28 \pm 0.14$ & $3.32 \pm 0.18$ \\
\hline $18: 4 n-1$ & $36 \pm 0.11$ & - & $0.27 \pm 0.02$ & & & & & & & \\
\hline $20: 0$ & $18 \pm 0.17$ & $.03 \pm 0.01$ & - & 00 & $4 \pm 0.04$ & & \pm 0.01 & & $0.55 \pm 0$ & 0.03 \\
\hline $20: 1 n-15$ & $0.01 \pm 0.01$ & $03 \pm 0.02$ & - & $0.03 \pm 0.03$ & $5.59 \pm 0.19$ & $5.89 \pm 0.27$ & $6.54 \pm 0.07$ & 0.19 & $4.59 \pm 0.13$ & $4.18 \pm 0.14$ \\
\hline $20: 1 n-11$ & $0.01 \pm 0.01$ & \pm 0.04 & $0.05 \pm 0.0$ & & & & & & .05 & \\
\hline $20: 1 n-9$ & $0.01 \pm 0.00$ & $0.04 \pm 0.04$ & $1 \pm 0.00$ & $0.02 \pm 0.02$ & \pm 1.00 & & $9 \pm 0.06$ & & $3.98 \pm 0.14$ & 0.20 \\
\hline $20: 1 n-7$ & - & $0.14 \pm 0.14$ & - & - & $4.55 \pm 0.13$ & $4.20 \pm 0.17$ & $4.64 \pm 0.13$ & $3 \pm 0.17$ & $3.92 \pm 0.13$ & $4.06 \pm 0.15$ \\
\hline & - & - & - & - & & & & & & \\
\hline $20: 2 \Delta 5,13$ & - & - & - & - & & & $3 \pm 0.10$ & & $9 \pm 0.15$ & 0.11 \\
\hline $20: 2 n-9$ & - & - & - & - & $0.25 \pm 0.03$ & $1 \pm 0.01$ & $23 \pm 0.01$ & $2 \pm 0.02$ & $0.18 \pm 0.01$ & $0.24 \pm 0.01$ \\
\hline $20: 1^{\mathrm{a}}$ & - & - & - & - & & & & & & \pm 0.04 \\
\hline & $.04 \pm 0.01$ & $48 \pm 0.14$ & $.03 \pm 0.01$ & $14+0$ & & & & & 04 & \\
\hline $20: 3 n-6$ & $0.41 \pm 0.19$ & $1.47 \pm 0.03$ & $0.29 \pm 0.01$ & $0.35 \pm 0.05$ & $6 \pm 0.03$ & $3 \pm 0.02$ & $0.41 \pm 0.00$ & $4 \pm 0.02$ & $0.61 \pm 0.03$ & $0.64 \pm 0.02$ \\
\hline & $3.90 \pm 0.48$ & $10.86 \pm 0.29$ & $8.09 \pm 0.29$ & $15.11 \pm 0.88$ & & & $5.39 \pm 0.09$ & & & $8 \pm 0.15$ \\
\hline & - & - & - & - & & & $5 \pm 0.03$ & 1.5 & $1.87 \pm 0.06$ & 0.05 \\
\hline $20: 3 n-3$ & $0.06 \pm 0.01$ & $11 \pm 0.00$ & $.09 \pm 0.01$ & $0.09 \pm 0.05$ & $1 \pm 0.06$ & & $0.83 \pm 0.01$ & $0.85 \pm 0.06$ & $0.76 \pm 0.03$ & $0.96 \pm 0.06$ \\
\hline $20: 4 n-3$ & $0.17 \pm 0.03$ & $0.31 \pm 0.01$ & $0.95 \pm 0.04$ & & & & $0.51 \pm 0.01$ & & $0.65 \pm 0.04$ & $0.95 \pm 0.05$ \\
\hline $20: 5 n-3$ & $8.80 \pm 0.13$ & $27.93 \pm 3.19$ & $17.35 \pm 0.70$ & $14.38 \pm 0.35$ & & & $5.65 \pm 0.10$ & $6.01 \pm 0.19$ & $9.00 \pm 0.46$ & $.04 \pm 0.28$ \\
\hline $22: 1 \mathrm{n}-11$ & - & - & - & - & $0.09 \pm 0.01$ & $0.11 \pm 0.02$ & $0.11 \pm 0.00$ & $0.10 \pm 0.02$ & $0.20 \pm 0.02$ & $0.10 \pm 0.01$ \\
\hline $22: 1 n-9$ & - & - & - & - & $2.95 \pm 0.16$ & $3.11 \pm 0.28$ & $3.22 \pm 0.04$ & $2.27 \pm 0.19$ & $2.90 \pm 0.16$ & $2.35 \pm 0.12$ \\
\hline $22: 1 n-7$ & - & - & - & - & & & $0.20 \pm 0.01$ & $0.14 \pm 0.01$ & $0.15 \pm 0.01$ & $0.14 \pm 0.01$ \\
\hline $22: 2 \Delta 7,13$ & - & - & - & - & & & & & $0.39 \pm 0.02$ & $0.47 \pm 0.02$ \\
\hline $22: 2 \Delta 7,15$ & - & - & - & - & $1.67 \pm 0.04$ & $1.88 \pm 0.11$ & $1.86 \pm 0.02$ & $1.33 \pm 0.08$ & $1.69 \pm 0.09$ & $1.62 \pm 0.14$ \\
\hline $22: 4 n-6$ & $0.10 \pm 0.0$ & 1 & $1 \pm 0$ & & & & $0.27 \pm 0.01$ & & $0.50 \pm 0.03$ & $0.36 \pm 0.02$ \\
\hline & $1+0$ & & $4 \pm 0.31$ & & & & & & $0.27 \pm 0.03$ & $0.17 \pm 0.01$ \\
\hline $22: 5 n-3$ & $11.21 \pm 2.99$ & $1.38 \pm 0.27$ & $0.02 \pm 0.01$ & $0.08 \pm 0.06$ & $0.26 \pm 0.10$ & $0.27 \pm 0.03$ & $0.30 \pm 0.01$ & $0.32 \pm 0.03$ & $0.46 \pm 0.03$ & $0.32 \pm 0.02$ \\
\hline & $0.04 \pm 0.01$ & $0.28 \pm 0.03$ & $0.27 \pm 0.05$ & $0.01 \pm 0.00$ & $0.73 \pm 0.13$ & $1.07 \pm 0.12$ & $1.08 \pm 0.03$ & $0.63 \pm 0.09$ & $1.78 \pm 0.13$ & $1.22 \pm 0.10$ \\
\hline
\end{tabular}




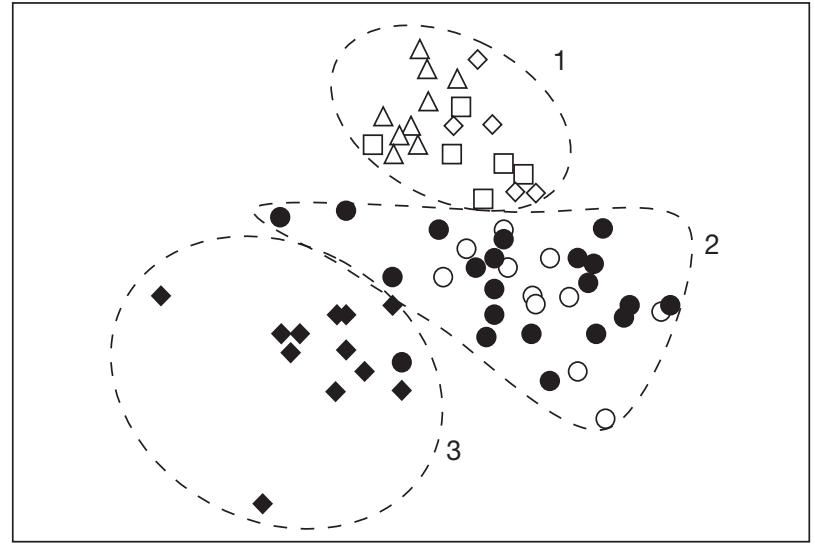

Fig. 2. Strongylocentrotus droebachiensis. Multidimensional scaling (MDS) plot of Bray-Curtis similarities of fatty acid (FA) compositions of sea urchins in 6 diet/habitat groups: urchins fed Agarum clathratum ( $\diamond)$, coralline algae ( $\square)$, Desmarestia viridis $(\Delta)$, and Saccharina longicruris $(\mathrm{O})$ in the laboratory, and wild urchins from barrens $(\bullet)$ and a kelp feeding front $(\bullet)$. Broken lines (---) indicate homogenous Clusters 1, 2 and 3 as determined by ANOSIM pairwise comparisons (Bonferroni adjusted $\alpha=0.05$ ) urchins from the kelp bed and urchins fed Saccharina longicruris in the laboratory; and (3) wild collected urchins from the barrens (Fig. 2). Within group similarity for these clusters was 91, 89 and $90 \%$, respectively.

Average dissimilarity was $13.5 \%$ between Clusters 1 and 2, $15.7 \%$ between Clusters 1 and 3, and $15.8 \%$ between Clusters 2 and 3. The FAs that contributed most to dissimilarity among clusters included saturated FAs (14:0 and 16:0), monounsaturated FAs (16:1, 16:1n-7, 20:1n-15, 20:1n-9), polyunsaturated FAs (16:4n-1, $18: 4 n-3,20: 4 n-6,20: 5 n-3)$ and non-methylene interrupted dienes $(20: \Delta 5,11$ and 20:2 $\Delta 5,13)$ (Table 2). Cluster 1 urchins were relatively rich in 20:1n-15,

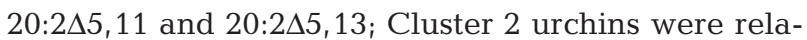
tively rich in 14:0 and 16:0, and Cluster 3 urchins were relatively rich in 16:4n-1 and 20:5n-3 (Table 2).

\section{DISCUSSION}

The FA compositions of the 4 algal taxa in this study were distinct (Table 1), and generally similar to those

Table 2. Strongylocentrotus droebachiensis. SIMPER analysis for relative abundance of fatty acids (mean \pm SE) in 3 clusters defined by ANOSIM pairwise comparisons. Cluster 1 contains urchins fed Agarum clathratum, Desmarestia viridis or coralline algae in the laboratory; Cluster 2 contains wild urchins from the kelp bed and urchins fed Saccharina longicruris in the laboratory; and Cluster 3 contains wild urchins from the barrens. Avg. = average Bray-Curtis dissimilarity; $(\%)=$ cumulative percentage of dissimilarity

\begin{tabular}{|c|c|c|c|c|c|c|}
\hline \multirow[t]{2}{*}{ Clusters } & \multirow[t]{2}{*}{ Fatty acid } & \multicolumn{3}{|c|}{ - Relative abundance- } & \multicolumn{2}{|c|}{ Dissimilarity } \\
\hline & & Cluster 1 & Cluster 2 & Cluster 3 & Avg. & $(\%)$ \\
\hline \multirow[t]{8}{*}{1 and 2} & $14: 0$ & $9.33 \pm 0.29$ & $12.57 \pm 0.36$ & & 1.7 & 12.5 \\
\hline & $16: 0$ & $12.07 \pm 0.18$ & $14.26 \pm 0.20$ & & 1.1 & 20.7 \\
\hline & $20: 1 n-15$ & $6.11 \pm 0.15$ & $4.30 \pm 0.12$ & & 0.9 & 27.4 \\
\hline & $20: 4 n-6$ & $5.07 \pm 0.21$ & $4.30 \pm 0.21$ & & 0.6 & 32.2 \\
\hline & $16: 1 n-7$ & $5.90 \pm 0.18$ & $5.71 \pm 0.26$ & & 0.6 & 36.6 \\
\hline & $20: 2 \Delta 5,11$ & $5.51 \pm 0.18$ & $4.56 \pm 0.11$ & & 0.6 & 40.9 \\
\hline & $20: 5 n-3$ & $5.41 \pm 0.16$ & $3.45 \pm 0.19$ & & 0.6 & 45.1 \\
\hline & $20: 1 n-9$ & $4.57 \pm 0.28$ & $2.80 \pm 0.16$ & & 0.6 & 49.1 \\
\hline \multirow[t]{9}{*}{1 and 3} & $20: 5 n-3$ & $5.41 \pm 0.16$ & & $9.00 \pm 0.46$ & 1.8 & 11.5 \\
\hline & $20: 2 \Delta 5,11$ & $5.51 \pm 0.18$ & & $2.68 \pm 0.15$ & 1.4 & 20.6 \\
\hline & $16: 4 n-1$ & $0.17 \pm 0.03$ & & $1.76 \pm 0.29$ & 0.8 & 25.7 \\
\hline & $20: 1 n-15$ & $6.11 \pm 0.15$ & & $4.59 \pm 0.13$ & 0.8 & 30.6 \\
\hline & $14: 0$ & $9.33 \pm 0.29$ & & $9.66 \pm 0.33$ & 0.7 & 25.1 \\
\hline & 16:0 & $12.07 \pm 0.18$ & & $13.14 \pm 0.30$ & 0.7 & 39.5 \\
\hline & $20: 2 \Delta 5,13$ & $3.77 \pm 0.15$ & & $2.59 \pm 0.15$ & 0.6 & 43.5 \\
\hline & 20:1n-9 & $4.57 \pm 0.28$ & & $3.98 \pm 0.14$ & 0.6 & 47.0 \\
\hline & $16: 1$ & $3.14 \pm 0.16$ & & $2.18 \pm 0.15$ & 0.5 & 50.5 \\
\hline \multirow[t]{9}{*}{2 and 3} & $14: 0$ & & $12.57 \pm 0.36$ & $9.66 \pm 0.33$ & 1.5 & 9.8 \\
\hline & $20: 5 n-3$ & & $6.03 \pm 0.19$ & $9.00 \pm 0.46$ & 1.5 & 19.4 \\
\hline & $20: 2 \Delta 5,11$ & & $4.56 \pm 0.11$ & $2.68 \pm 0.15$ & 0.9 & 25.3 \\
\hline & $16: 4 n-1$ & & $0.26 \pm 0.03$ & $1.76 \pm 0.29$ & 0.8 & 30.2 \\
\hline & 16:0 & & $14.26 \pm 0.20$ & $13.14 \pm 0.30$ & 0.8 & 35.0 \\
\hline & $16: 1$ & & $3.48 \pm 0.14$ & $2.18 \pm 0.15$ & 0.7 & 39.3 \\
\hline & $16: 1 \mathrm{n}-7$ & & $5.71 \pm 0.26$ & $5.94 \pm 0.27$ & 0.6 & 43.2 \\
\hline & $20: 4 n-6$ & & $4.30 \pm 0.21$ & $4.63 \pm 0.30$ & 0.6 & 47.2 \\
\hline & $18: 4 n-3$ & & $3.45 \pm 0.13$ & $2.28 \pm 0.14$ & 0.6 & 51.1 \\
\hline
\end{tabular}


recorded in previous studies of the same or congeneric species: Corallina pilulifera (Kitamura et al. 1993), Agarum cribosum (= clathrathum) (Khotimchenko 1998), and Saccharina longicruris (Cook et al. 2000). The FA composition of Desmarestia viridis in our study differed from that reported by Li et al. (2002) in that it was lower in 16:0 and 18:1n-9. Likewise, urchins fed different algal diets had distinct gonadal FA compositions; however, these did not directly correspond to those of their diets. Substantial levels of non-methylene interrupted dienes and other FAs present in urchin gonads, but not in their diets, as well as higher levels of certain FAs in urchin gonads than in algae, indicate significant levels of FA biosynthesis in urchins. This level of biosynthesis clearly reduced the influence of dietary FAs, although the urchins were still separated by their dietary groups. Although the gonads are the key site of lipid storage in sea urchins, the gonads also have a dual function as a reproductive organ and may therefore experience greater biochemical and FA modification than do other types of lipid storage areas (Hughes et al. 2006). Thus, although other invertebrate herbivores may also synthesize significant amounts of FAs, these effects may be particularly pronounced in urchins due to their specific site of lipid storage.

Although a correspondence between the FA compositions of urchin gonads and their diets has been documented in other studies (Cook et al. 2000, LiyanaPathirana et al. 2002, Castell et al. 2004), these have compared a single algal diet to various synthetic diets of markedly different compositions. Urchins convert some dietary FAs to new FA products through various biosynthetic pathways (Cook et al. 2000, Castell et al. 2004). While synthesized FAs cannot be used as direct dietary indicators, their levels may vary with diet. For example, Castell et al. (2004) found correlations between dietary levels of precursor FAs (18:2n-6 and 18:3n-3) and levels of synthesized FAs (20:4n-6, 20:5n-3, 22:6n-3 and 20:2 $\Delta 5,11)$ in urchin gonads. We did not find such correlations between dietary precursors and products of biosynthesis in urchins.

Gonads of urchins fed Agarum clathratum, coralline algae and Desmarestia viridis in the laboratory contained relatively high amounts of the urchin synthesized FAs: $20: 2 \Delta 5,11$ and 20:2 $\Delta 5,13$. The urchins fed these 3 non-kelp algal diets had low estimated intake rates, the lowest fat contents in their gonads (Fig. 1), and clustered together in MDS analysis (Fig. 2). The effect of a particularly low fat-intake diet should stimulate FA biosynthetic rates, possibly contributing further to the low apparent influence of dietary FAs. However, although the urchins fed kelp in the laboratory and those collected from kelp grazing fronts formed a separate cluster in MDS analysis, there was no direct concordance between FAs in urchin gonads and their kelp diet (Table 1).

The gonad FAs of urchins from barrens differed from those of all other groups, suggesting that the characteristic macroalgae of urchin barrens in eastern Canada-Agarum clathratum, coralline algae and Desmarestia viridis, which are all chemically or mechanically defended against urchin grazing (Himmelman \& Nédélec 1990, Gagnon et al. 2003, 2005)—do not contribute significantly to the diet of urchins in this habitat. The polyunsaturates 16:4n-1 and 20:5n-3 were important in separating barrens urchins from all others, and accounted for an average of $1.8 \%$ and $9.0 \%$, respectively, of total FAs in these urchins. The presence of these FAs suggests that urchins derive some FAs from microalgal films growing on coralline algae in barrens, as 16:4n-1 and 20:5n-3 are abundant in diatoms (Sargent et al. 1988). Diatoms and filamentous brown and red algae have been found in the gut of urchins from barrens in Nova Scotia (Chapman 1981).

Wild urchins from a grazing front at the lower margin of a kelp bed and urchins fed Saccharina longicruris in the laboratory had similar gonad FA compositions, indicating that urchins in fronts derive most of their nutrition from $S$. longicruris and similar kelp species. This is consistent with field observations of dense urchin fronts that rapidly consume all kelp in their path (Chapman 1981, Scheibling et al. 1999, Gagnon et al. 2004). Lauzon-Guay \& Scheibling (2007) estimated that individual urchins at the grazing front at Splitnose Point consumed kelp at a rate of $0.027 \mathrm{~g} \mathrm{~d}^{-1}$. Differences between the wild and laboratory urchins may be partly due to consumption of Laminaria digitata, which comprised approximately half of kelp biomass at this site (Lauzon-Guay \& Scheibling 2007). The gonad FA compositions of both wild urchins from a grazing front and urchins fed $S$. longicruris in the laboratory in this study were generally similar to FA signatures found by Liyana-Pathirana et al. (2002) and by Castell et al. (2004) using the same urchin and kelp species in controlled feeding experiments, and by Takagi et al. (1980) in wild urchins from a Nova Scotian kelp bed.

As stated previously, gonads of urchins fed single diets of the characteristic macroalgae of barrens had lower lipid contents than did gonads of wild urchins and of urchins fed Saccharina longicruris, suggesting that these barrens macroalgae are poor nutritional sources for urchins. Differences in sampling period between wild collected (November) and laboratory fed (September) urchins may account for some difference in lipid content between these groups, related to the gametogenic cycle and increasing gonad size in the fall (Meidel \& Scheibling 1998). However, the similarity in gonad FA signatures between urchins collected from the kelp grazing front and those fed S. longicruris 
in the laboratory suggests that, although seasonal differences could have affected gonad size and lipid content, they did not affect the FA composition. Gender also affects gonad FA composition of urchins (Hughes et al. 2006) and may account for some of the within group variability in our study.

We have shown that the FA composition of urchin gonads is substantially influenced by de novo FA synthesis and does not closely resemble that of their algal diets. Therefore, it is unlikely that algal FAs can be used as direct dietary tracers for sea urchins in the Nova Scotian system. However, our ability to distinguish urchins fed different algal diets by their gonad FA compositions indicates that FAs may serve as indirect dietary tracers in determining the source of primary production used by consumers at higher trophic levels. We also found evidence that urchins in barrens obtain much of their nutrition from nonmacroalgal sources such as diatoms, and that these sources may be more nutritious than macroalgae typically found in barrens such as Agarum clathratum, corallines and Desmarestia viridis. Captive feeding experiments that include algae, herbivores (including those that can synthesize a significant portion of their FAs) and higher order consumers are required to determine the applicability of FA analysis to bottom-up tracking of energy flow in benthic marine food webs.

Acknowledgements. We thank J. Lindley, J.-S. Lauzon-Guay, D. Lyons, L. Sauchyn, M. Saunders, and S. Watanabe for their assistance in the field, S. Al-Shaghay and S. Budge for analytical advice and laboratory support, and J. Jones and A. Young for their assistance in the laboratory. We also thank S. Budge and T. Romanuk for their comments on a draft of this manuscript. This research was funded by Discovery Grants and Research Tools and Instruments Grants to R.E.S and S.J.I. from the Natural Sciences and Engineering Research Council (NSERC) of Canada. J.R.K. was supported by a Dalhousie Scholarship, a Killam Predoctoral Scholarship and a NSERC Canada Graduate Scholarship.

\section{LITERATURE CITED}

Alfaro AC, Thomas F, Sergent L, Duxbury M (2006) Identification of trophic interactions within an estuarine food web (northern New Zealand) using fatty acid biomarkers and stable isotopes. Estuar Coast Shelf Sci 70:271-286

> Bottino NR (1974) The fatty acids of antarctic phytoplankton and euphausiids. Fatty acid exchange among trophic levels of the Ross Sea. Mar Biol 27:197-204

Budge SM, Iverson SJ, Bowen WD, Ackman RG (2002) Among- and within-species variability in fatty acid signatures of marine fish and invertebrates on the Scotian Shelf, Georges Bank, and southern Gulf of St. Lawrence. Can J Fish Aquat Sci 59:886-898

Budge SM, Iverson SJ, Koopman HN (2006) Studying trophic ecology in marine ecosystems using fatty acids: a primer on analysis and interpretation. Mar Mamm Sci 22:759-801

> Castell JD, Kennedy EJ, Robinson SMC, Parsons GJ, Blair TJ, Gonzalez-Duran E (2004) Effect of dietary lipids on fatty acid composition and metabolism in juvenile green sea urchins (Strongylocentrotus droebachiensis). Aquaculture 242:417-435

> Chapman ARO (1981) Stability of sea urchin dominated barren grounds following destructive grazing of kelp in St. Margaret's Bay, Eastern Canada. Mar Biol 62:307-311

Clarke KR, Warwick RM (1994) Change in marine communities: an approach to statistical analysis and interpretation. Natural Environment Research Council, London

Cook EJ, Bell MV, Black KD, Kelly MS (2000) Fatty acid compositions of gonadal material and diets of the sea urchin, Psammechinus miliaris: trophic and nutritional implications. J Exp Mar Biol Ecol 255:261-274

Dalsgaard J, St. John M, Kattner G, Muller-Navarra D, Hagen W (2003) Fatty acid trophic markers in the pelagic marine environment. Adv Mar Biol 46:225-340

Folch J, Lees M, Sloane Stanley GH (1957) A simple method for the isolation and purification of total lipids from animal tissues. J Biol Chem 226:497-509

Gagnon P, Himmelman JH, Johnson LE (2003) Algal colonization in urchin barrens: defense by association during recruitment of the brown alga Agarum cribrosum. J Exp Mar Biol Ecol 290:179-196

Gagnon P, Himmelman JH, Johnson LE (2004) Temporal variation in community interfaces: kelp-bed boundary dynamics adjacent to persistent urchin barrens. Mar Biol 144:1191-1203

Gagnon P, Johnson LE, Himmelman JH (2005) Kelp patch dynamics in the face of intense herbivory: stability of Agarum clathratum (Phaeophyta) stands and associated flora on urchin barrens. J Phycol 41:498-505

Graeve M, Kattner G, Hagen W (1994) Diet-induced changes in the fatty acid composition of Arctic herbivorous copepods: experimental evidence of trophic markers. J Exp Mar Biol Ecol 182:97-110

Graeve M, Kattner G, Wiencke C, Karsten U (2002) Fatty acid composition of Arctic and Antarctic macroalgae: indicator of phylogenetic and trophic relationships. Mar Ecol Prog Ser 231:67-74

Hall D, Lee SY, Meziane T (2006) Fatty acids as trophic tracers in an experimental estuarine food chain: tracer transfer. J Exp Mar Biol Ecol 336:42-53

Hayashi K, Takagi T (1977) Occurrence of branched- and oddchain fatty acids in a mud-feeding sea urchin, Strongylocentrotus franciscanus. Bull Fac Fish Hokkaido Univ 28: $40-46$

> Himmelman JH, Nédélec H (1990) Urchin foraging and algal survival strategies in intensely grazed communities in eastern Canada. Can J Fish Aquat Sci 47:1011-1026

> Hughes AD, Kelly MS, Barnes DKA, Catarino AI, Black KD (2006) The dual functions of sea urchin gonads are reflected in the temporal variations of their biochemistry. Mar Biol 148:789-798

Iverson SJ (1993) Milk secretion in marine mammals in relation to foraging: can milk fatty acids predict diet? Symp Zool Soc Lond 66:263-291

Iverson SJ (2009) Tracing aquatic food webs using fatty acids: from qualitative indicators to quantitative determination. In: Arts MT, Brett MT, Kainz M (eds) Lipids in aquatic ecosystems. Springer-Verlag, New York (in press)

> Iverson SJ, Frost KJ, Lowry LF (1997) Fatty acid signatures reveal fine scale structure of foraging distribution of harbor seals and their prey in Prince William Sound, Alaska. Mar Ecol Prog Ser 151:255-271

Iverson SJ, Field C, Bowen WD, Blanchard W (2004) Quantitative fatty acid signature analysis: a new method of estimating predator diets. Ecol Monogr 74:211-235 
Iverson SJ, Springer AM, Kitaysky AS (2007) Seabirds as indicators of food web structure and ecosystem variability: qualitative and quantitative diet analyses using fatty acids. Mar Ecol Prog Ser 352:235-244

Johnson CR, Mann KH (1982) Adaptations of Strongylocentrotus droebachiensis for survival on barren grounds in Nova Scotia. In: Lawrence JM (ed) Echinoderms: Proc Int Conf, Tampa Bay. A.A. Balkema, Rotterdam, p 277-283

Kharlamenko VI, Kiyashko SI, Imbs AB, Vyshkvartzev DI (2001) Identification of food sources of invertebrates from the seagrass Zostera marina community using carbon and sulfur stable isotope ratio and fatty acid analyses. Mar Ecol Prog Ser 220:103-117

Khotimchenko SV (1998) Fatty acids of brown algae from the Russian Far East. Phytochemistry 49:2363-2369

Khotimchenko SV (2003) Fatty acids of species in the genus Codium. Bot Mar 46:456-460

Khotimchenko SV, Vaskovsky VE, Titlyanova TV (2002) Fatty acids of marine algae from the Pacific coast of North California. Bot Mar 45:17-22

Kirsch PE, Iverson SJ, Bowen DW, Kerr SR, Ackman RG (1998) Dietary effects on the fatty acid signature of whole Atlantic cod (Gadus morhua). Can J Fish Aquat Sci 55: 1378-1386

> Kitamura H, Kitahara S, Koh HB (1993) The induction of larval settlement and metamorphosis of two sea urchins, Pseudocentrotus depressus and Anthocidaris crassispina, by free fatty acids extracted from the coralline red alga Corallina pilulifera. Mar Biol 115:387-392

Lauzon-Guay JS, Scheibling RE (2007) Behaviour of sea urchin Strongylocentrotus droebachiensis grazing fronts: food-mediated aggregation and density-dependent facilitation. Mar Ecol Prog Ser 329:191-204

Li X, Fan X, Han L, Lou Q (2002) Fatty acids of some algae from the Bohai Sea. Phytochemistry 59:157-161

Liyana-Pathirana C, Shahidi F, Whittick A (2002) The effect of an artificial diet on the biochemical composition of the gonads of the sea urchin (Strongylocentrotus droebachiensis). Food Chem 79:461-472

Meidel SK, Scheibling RE (1998) Annual reproductive cycle of the green sea urchin, Strongylocentrotus droebachiensis, in differing habitats in Nova Scotia, Canada. Mar Biol 131: 461-478

Meziane T, d'Agata F, Lee SY (2006) Fate of mangrove organic matter along a subtropical estuary: small-scale exportation and contribution to the food of crab communities. Mar Ecol Prog Ser 312:15-27

Nelson MM, Phleger CF, Nichols PD (2002) Seasonal lipid composition in macroalgae of the Northeastern Pacific Ocean. Bot Mar 45:58-65

Editorial responsibility: Hans Heinrich Janssen, Oldendorf/Luhe, Germany
Nichols PD, Klumpp DW, Johns RB (1986) Lipid components and utilization in consumers of a seagrass community: an indication of carbon source. Comp Biochem Physiol B Biochem Mol Biol 83:103-113

Nordstrom CA, Wilson LJ, Iverson SJ, Tollit DJ (2008) Evaluating quantitative fatty acid signature analysis (QFASA) using harbour seals Phoca vitulina richardsi in captive feeding studies. Mar Ecol Prog Ser 360:245-263

Pantazis PA, Kelly MS, Connolly JG, Black KD (2000) Effect of artificial diets on growth, lipid utilization, and gonad biochemistry in the adult sea urchin Psammechinus miliaris. J Shellfish Res 19:995-1001

Parrish CC, Abrajano TA, Budge SM, Helleur RJ, Hudson ED, Pulchan K, Ramos C 2000. Lipid and phenolic biomarkers in marine ecosystems: analysis and applications. In: Wangersky P (ed) The handbook of environmental chemistry, Part D-marine chemistry. Springer, Berlin, p 193-222

Pond DW, Priddle J, Sargent JR, Watkins JL (1995) Laboratory studies of assimilation and egestion of algal lipid by Antarctic krill-methods and initial results. J Exp Mar Biol Ecol 187:253-268

> Reuss N, Poulsen LK (2002) Evaluation of fatty acids as biomarkers for a natural plankton community. A field study of a spring bloom and a post-bloom period off West Greenland. Mar Biol 141:423-434

> Rooker JR, Turner JP, Holt SA (2006) Trophic ecology of Sargassum-associated fishes in the Gulf of Mexico determined from stable isotopes and fatty acids. Mar Ecol Prog Ser 313:249-259

Sargent JR, Parkes RJ, Mueller-Harvey I, Henderson RJ (1988) Lipid biomarkers in marine ecology. In: Sleigh MA (ed) Microbes in the sea. Ellis Horwood, Chichester, p 119-183

Scheibling RE (1986) Increased macroalgal abundance following mass mortalities of sea urchins (Strongylocentrotus droebachiensis) along the Atlantic coast of Nova Scotia. Oecologia 68:186-198

Scheibling RE, Hatcher BG (2007) Ecology of Strongylocentrotus droebachiensis. In: Lawrence JM (ed) Edible sea urchins: biology and ecology. Elsevier, Amsterdam, p 353-392

> Scheibling RE, Hennigar AW, Balch T (1999) Destructive grazing, epiphytism, and disease: the dynamics of sea urchin-kelp interactions in Nova Scotia. Can J Fish Aquat Sci 56:2300-2314

- Takagi T, Eaton CA, Ackman RG (1980) Distribution of fatty acids in lipids of the common Atlantic sea urchin Strongylocentrotus droebachiensis. Can J Fish Aquat Sci 37: 195-202

Submitted: July 22, 2008; Accepted: September 18, 2008

Proofs received from author(s): November 22, 2008 